Stufe 3 schließlich besteht im Informationsaustausch mit umweltmedizinischen Einrichtungen und hinreichender Information aus anderen Quellen. Hinzu kommen Schadstoffmessungen sowohl in Blut- und Harnproben des Patienten (Bio-Monitoring) als auch in der Innenraumluft (Umwelt-Monitoring). Für wichtig hält es Frau Voack, dabei nur mit zuverlässigen Labors zusammenzuarbeiten und nur Messungen $\mathrm{zu}$ veranlassen, die man auch interpretieren kann.

Ebenso wichtig ist die kritische Interpretation der Befunde. Die Überschreitung eines Grenzwertes ist nicht unbedingt mit einer gesundheitlichen Störung oder Schädigung gleichzusetzen, sie ist lediglich ein Hinweis auf eine erhöhte Exposition. „Nicht der Meßwert ist entscheidend, sondern die individuelle Interpretation", betonte Voack.

Stufe 4 schließlich umfaßt abschließende Diagnosestellung, Risikoabschätzung und therapeutische Konsequenzen. Hier kommt es vor allem auf die Verlaufsbeobachtung an. Von ihr hängt es $a b$, ob eine vorhandene Schadstoffbelastung wirklich die Gesundheit gefährdet. Eine individuelle
Beratung und Betreuung sind notwendig, eventuell eine psychosomatischpsychiatrische Begleitung, ebenso aber auch geeignete präventivmedizinische Maßnahmen.

\section{Großer Forschungsbedarf}

Auf die Tatsache, daß Innenraumschadstoffe auch allergologische Erkrankungen verschlimmern können, wies B. Eberlein-König, Klinik und Poliklinik für Dermatologie und Allergologie am Biederstein, TU München, hin. Unter den nichtbiologischen Bestandteilen der Innenraumluft ist es vor allem das $\mathrm{SO}_{2}$, das allein oder in Kombination mit Allergenen ein atopisches Ekzem, eine Rhinoconjunctivitis allergica bzw. die Lungenfunktionsparameter bei Patienten mit Asthma bronchiale erwiesenermaßen verschlechtern kann. Auch für Formaldehyd gibt es Befunde, die eine Verschlechterung von Rhinitis allergica und atopischem Ekzem belegen. Insgesamt besteht, wie Eberlein-König betonte, auf dem Gebiet noch erheblicher Forschungsbedarf.

(ue)

Nach Vorträgen beim Kongreß „Fortschritte der Allergologie, Immunologie und Dermatologie“, Davos, 10. bis 14 September 1997.

\title{
Medikamente gegen Allergien: Billig kann teuer kommen
}

D er ungebrochene Anstieg allergischer Erkrankungen bei eng begrenzten Ressourcen zwingt bei der Behandlung $\mathrm{zu}$ einer strengen Kosten-Nutzen-Analyse. Die freilich zeigt, daß die preiswerteste Therapie stets diejenige ist, die das Fortschreiten der Erkrankung und den „Etagenwechsel" am wirksamsten verhindert.

Vom Gesamtbudget für die Behandlung der Inhalationsallergie entfallen laut F. Horak, UniversitätsHNO-Klinik Wien, rund zwei Drittel auf die Asthmatherapeutika, das restliche Drittel etwa zu gleichen Teilen auf Rhinologika, Antihistaminika und Substanzen zur Hyposensibilisierung. $\mathrm{Zu}$ den Krankheitskosten hinzugerechnet werden müssen nicht nur Arzthonorare und Krankenhauskosten, sondern auch die indirekten Kosten, etwa durch Arbeitsausfall.

Will man mit dem Sparen bei den Medikamenten beginnen, kann ein Preisvergleich in die Irre führen. Oft werden Dosierungen angegeben, die sich in der Praxis als unzureichend erweisen. Stellt man dann die optimalen Mengen gegenüber, fällt die Rangordnung der Preiswürdigkeit oft ganz anders aus als auf den ersten Blick, so Horaks Erfahrung.

Auch der Patient mit saisonaler allergischer Rhinitis, der den Arzt übergeht und sich selbst ein Medikament besorgt, spart nicht unbedingt wirklich. Wählt er eine stark sedierende, seine Leistungsfähigkeit drastisch einschränkende Substanz, kommt die Sparsamkeit teuer. Wird aus der allergischen Rhinitis unter suboptimaler Behandlung ein allergisches Asthma, wird es erst richtig kostspielig. Direkte und indirekte Kosten des Asthmas steigen mit dem Schweregrad und stehen denen des Herzinfarkts in Deutschland nur wenig nach.

„Es liegt an uns“, betonte Horak. „Je weniger Patienten den ,Etagenwechsel' durchmachen, desto kostengünstiger sind wir - auch wenn das die Kostenträger oft nicht akzeptieren wollen."

\section{Hyposensibilisierung rechnet sich}

Ein nachweislich bewährter Weg, die Komplikation Asthma zu verhüten, ist die Hyposensibilisierung. Angewandt wird sie jedoch immer noch sehr selten, beklagte Horak. Hier sind die Unterschiede in Europa riesig. In Skandinavien werden rund 600 von 1 Million Einwohnern behandelt, in Spanien mehr als 10400 pro 1 Million. Deutschland liegt mit einer Rate von 6500 pro 1 Million etwa im Mittelfeld. Extrem selten allerdings wird in Deutschland bei Insektengiftallergien desensibilisiert (8\%, gegenüber $32 \%$ in Skandinavien).

Die anfänglich im Vergleich zur symptomatischen Therapie höheren Kosten der Hyposensibilisierung amortisieren sich nach wenigen Jahren, was insbesondere bei Pollenallergien gilt. Ein Kostenvergleich bei den verfügbaren Präparaten lohnt, so Horak. Ob auch die Hyposensibilisierung bei Hausstaubmilbenallergien als kosteneffektiv gelten kann, wird allerdings kontrovers beurteilt.

(ue)

Nach einem Vortrag beim Kongreß „Fortschritte der Allergologie, Immunologie und Dermatologie“, Davos, 10. bis 14 September 1997 